\title{
Cachez cette réforme que je ne saurais voir. Le cas des compléments du verbe
}

\author{
Emilie Deschellette, et Audrey Roig \\ Université Paris Descartes, EDA (EA 4071) \\ emilie.deschellette@parisdescartes.fr
}

\begin{abstract}
Résumé. La disparition des adjectifs «direct» et «indirect» qui accompagnaient jusqu'il y a peu les compléments $d u$ verbe dans la terminologie grammaticale pour l'école en France ( $c f$. Bulletin officiel du 26 novembre 2015, présentant les nouveaux contenus scolaires) ne va pas sans appeler une question plus large: celle de la prédisposition des professeurs du premier et second degré à enseigner un discours grammatical réformé. À travers l'étude du cas du complément d'objet indirect $(\mathrm{COI})$, parangon de la récente réforme du discours grammatical, cet article entend observer les représentations de la grammaire et de la terminologie chez une centaine d'enseignants actuellement en formation initiale. Il entend pareillement interroger une série d'élèves de CE2, CM2 et $3^{\mathrm{e}}$ en vue d'identifier la réception et la perception de ce discours par les élèves.
\end{abstract}

\begin{abstract}
The grammar doth protest too much, methinks. The case of "compléments du verbe" (objects of the verb). The replacement of the phrases "direct object" and "indirect object" by the brand new generic expression "complements of the verb" in one of the latest French school reforms (see Official Bulletin of $26^{\text {th }}$ November 2015, presenting the new school programs) raises a broader issue: the predisposition of teachers in primary school, middle school and high school to adopt and use a reformed grammatical discourse in their classrooms. Through the study of the Indirect Object ( $\mathrm{IO})$, a perfect example of the aforementioned reform, this article intends to investigate the mental representations of 161 teachers currently receiving initial training on the subject of grammar and terminology. This study also intends to analyze the answers of pupils in $3^{\text {rd }}$, $5^{\text {th }}$ and $9^{\text {th }}$ grade (=CE2,CM2 and $3^{e}$ in the French system) in order to identify the way they receive and perceive this discourse.
\end{abstract}

Les compléments d'objet direct (COD) et indirect (COI) sont loin d'être des créations récentes [1]. Hérités des deux cas différents en latin, l'accusatif et le datif, mais regroupés en ancien et moyen français sous la seule étiquette de "cas régime », les deux types de compléments vont progressivement être (re)distingués au fil du temps, en réponse notamment à une question d'ordre orthographique. En effet, comme le remarque Piron [2], si à l'aube du XVIII siècle "les grammaires traitent de plus en plus précisément les compléments du verbe et proposent une esquisse de terminologie pour ce type de complémentation », des auteurs comme Buffier [3] vont notamment suggérer d'opérer une 
distinction entre le régime absolu (ou direct, fruit de l'accusatif) et le régime respectif (ou indirect, fruit du datif) [4], utile pour le bon accord du participe passé. La porte entrouverte est alors rapidement enfoncée par des auteurs comme Restaut [5] puis Lhomond [6] qui sont parmi les pionniers à présenter des « questions pour trouver le complément direct, le complément indirect et le sujet » [7].

La publication de la grammaire de Noël et Chapsal [8] semble marquer un tournant majeur dans l'histoire de la complémentation. Emblème de la vague de la première grammaire scolaire [9], « La grammaire de Noël et Chapsal constitue le socle sur lequel se construira l'édifice de la théorie grammaticale scolaire. L'ouvrage est cautionné par l'Instruction publique et utilisé comme manuel dans les écoles » [10]. Associant encore l'analyse grammaticale à l'analyse logique, étroitement mêlées déjà au XVIII ${ }^{\mathrm{e}}$ siècle, la grammaire de Noël \& Chapsal présente clairement deux types de compléments, directs et indirects, rencontrés déjà chez Girault-Duvivier [11] au début du XIX ${ }^{\mathrm{e}}$ siècle. Noël \& Chapsal [8] donnent cependant une définition très large de ces compléments : qu'ils soient directs ou indirects, les « deux compléments "complètent l'idée commencée par un autre mot" (LAG, p. 4)» [10], peu importe la nature de cet autre mot.

Un mot qui occupe la fonction de complément direct se repère au moyen de la question, déjà traditionnelle au $\mathrm{XIX}^{\mathrm{e}}$ siècle, qui ? ou quoi ? Ce complément est directement lié au mot qui le précède, sans aucune préposition. À la différence du complément direct, le complément indirect introduit un complément au moyen d'une préposition quelconque ( $a$, de, par, pour, etc.). Les questions traditionnelles servent à le repérer facilement : à qui ? de $q u i$ ? etc. La grande différence que perçoit immédiatement un lecteur moderne réside dans le fait que le complément indirect de la théorie chapsalienne peut se raccrocher à n'importe quel mot pouvant être suivi d'une préposition. Il est ainsi possible de trouver un complément indirect d'un substantif (la maison de votre père), d'un pronom (celle de votre frère), d'un adjectif (digne de louanges), d'un adverbe (beaucoup d'esprit) et, bien sûr, d'un verbe (parler à quelqu'un, et même travailler pour la gloire) [10].

$\mathrm{C}^{\prime}$ est au début du $\mathrm{XX}^{\mathrm{e}}$ siècle que les notions grammaticales de compléments direct et indirect se verront restreintes au domaine verbal, en vue de les distinguer clairement des compléments circonstanciels qui peuvent pareillement être introduits, parfois, par des prépositions (La nuit, les enfants dorment; Il sort à la tombée de la nuit). L'analyse grammaticale prenant le pas sur l'analyse logique, c'est l'ensemble des fonctions qui est alors redéfini, et en regard de la place toujours croissante laissée à l'orthographe (et à l'accord du participe passé), il apparait essentiel de distinguer les COD des COI, la distinction se faisant d'après la présence ou non d'une préposition. « Si la terminologie a hésité quelque temps entre complément d'objet (in)direct et complément (in)direct d'objet, la première dénomination l'a emporté parce que la notion d'objet a gagné en importance et subsume la construction directe ou indirecte » [12]. La distinction s'est conservée depuis lors, et elle est retrouvée à l'identique dans les programmes scolaires français du B.O. du 19 juin 2008 pour l'école primaire (cycle 3) à travers l'entrée "compléments du verbe : compléments d'objet direct, indirect et second» ([13], p. 22), du 28 aout 2008 pour le collège (cycle 4) à travers la mention des « compléments d'objet du verbe (direct, indirect, second) » ([14], p. 6). Les notions de COD et de COI sont rencontrées à l'identique dans le Code de terminologie français de 1997 [15] aux côtés des compléments dits « essentiels » du verbe («Compléments essentiels exprimant le lieu, le prix, le poids, la mesure, la durée, etc. »; [15], p. 17). D’emblée, les compléments essentiels et seconds paraissent mal établis, à telle enseigne qu'ils ont disparu des programmes officiels français qui entreront en vigueur à la rentrée 2016 [16]. En effet, seule l'entrée «complément du verbe » est présentée dans l'encart terminologique au cycle 3 comme au cycle 4 dans les programmes du 26 novembre 2015 en France ([16], p. 118, 245), sans distinction entre direct et indirect. La mention objet a elle aussi été supprimée. L'on constate en définitive le passage du rasoir 
d'Occam sur la terminologie en vigueur depuis peu, lequel procède d'une conception de l'étude de la langue orientée vers une simplification du discours grammatical. Cette façon d'envisager la grammaire conduit à l'élimination d'une série de fioritures métalinguistiques pour mettre l'accent sur des distinctions plus génériques et plus fondamentales telles que celle qui oppose les compléments du verbe aux compléments phrase (ou circonstanciels) sur la base de trois manipulations syntaxiques : la suppression, le déplacement en tête de phrase et la pronominalisation ([16], cycle 3, p. 120). Cet exemple est, selon nous, représentatif de la mise en place d'une nouvelle approche du traitement et de l'enseignement de la grammaire.

Passé la houle suscitée par la publication du Bulletin officiel du 26 novembre 2015 [16] présentant les nouveaux contenus scolaires français, il nous a semblé judicieux de revenir sur le cas du COI comme parangon d'une problématique plus vaste, celle de la prédisposition des professeurs du premier et second degré à enseigner un discours grammatical réformé. Plus précisément, les questions que nous nous sommes posées entendaient revenir sur trois problématiques principales : (a) la pertinence des contenus grammaticaux - qui, en regard des nouvelles directives ministérielles, sont représentés ici par le questionnement des compléments verbaux, (b) la disposition des enseignants à embras(s)er une nouvelle terminologie grammaticale en amont, un système d'analyse différent en aval, (c) le statut de l'étude de la langue à l'école et au collège, aux yeux à la fois des élèves et des (futurs) enseignants en France.

\section{Présentation de l'enquête}

Pour répondre à ces questions, nous avons choisi de sonder différentes personnes, sélectionnées pour leur profil hétérogène, notamment des enseignants actuellement en formation initiale, à dessin d'observer l'écho de leurs représentations de la grammaire et de la terminologie. Nous avons pareillement interrogé une série d'élèves de CE2, CM2 ${ }^{1}$ et $3^{\mathrm{e} 2}$ en vue d'identifier leur réception du discours enseigné.

Les questionnaires ${ }^{3}$ soumis aux élèves présentaient quatre questions quand ceux remis aux futurs enseignants en comportaient six : les quatre premières sont communes à celles posées aux élèves, les deux autres visaient à mieux comprendre le regard que les enseignants portaient sur le discours grammatical et sa transposition didactique.

1) a. Aimez-vous l'étude de la langue ? $\square$ oui $\square$ non

b. Pour quelles raisons?

2) Cochez toutes les propositions qui vous semblent définir le COI.

Le « COI» :

$\square$ est la même chose que le COD.

$\square$ est le complément d'objet indirect de la phrase.

$\square$ est un groupe qui peut être déplacé dans la phrase.

$\square$ est le plus souvent introduit par les prépositions $a ̀$ ou $d e$.

est identifié au moyen de la question «Qui ? » ou «Quoi ? ».

$\square$ donne des indications de temps, lieu, manière sur l'action qui se déroule. 
3) Cochez les phrases qui ont un COI et soulignez-le dans la phrase.

$\square$ Julien attend son frère.

$\square$ Le jeune homme succédera à son père l'an prochain.

$\square$ Pense aux sans abris.

$\square$ Juliette va à la piscine le mercredi matin.

$\square$ Il se souvient de sa première bicyclette.

$\square$ Les enfants se donnent la main.

$\square$ J'y pense.

4) Selon vous, à quoi cela sert-il de chercher à identifier le «COI» dans une phrase ?

Questions ajoutées pour les enseignants :

5) Vous semble-t-il pertinent de le distinguer du COD ? Justifiez votre réponse.

6) a. Seriez-vous favorable à ne plus différencier les COD et COI, pour ne plus parler que de « compléments du verbe »(sans distinction)? $\square$ oui $\square$ non

b. Pour quelles raisons?

La première question entendait sonder le gout plus ou moins prononcé des élèves et des enseignants pour l'étude de la langue en général, ainsi que les motifs de leur (non-)appréciation.

La deuxième question, qui allait de pair avec la troisième, s'attardait sur la connaissance théorique du « COI » et de ses propriétés (aspect théorique); la troisième avait quant à elle pour objectif d'évaluer les compétences des enquêtés en matière de reconnaissance du COI dans une phrase (aspect pratique).

Inscrite dans le prolongement des deux précédentes, la quatrième question interrogeait les élèves et futurs enseignants sur l'utilité qu'ils reconnaissent à l'activité d'identification d'un COI dans une phrase : à quoi cela sert-il de trouver le COI dans un énoncé ?

Les cinquième et sixième étaient donc réservées au public plus âgé en ce qu'elles ont davantage trait à la problématique de la transposition didactique: que convient-il d'enseigner aux élèves ? Est-il pertinent d'apprendre à l'élève de différencier le COI du COD ? Et est-il favorable de ne plus les distinguer pour les rassembler sous la seule appellation de «complément du verbe » (comme le prônent d'ailleurs les textes officiels pour 2016 [16] ; cf. infra)?

Ces questions ancrent toutes leurs racines dans un questionnement plus général : dans quelles mesures le point de vue des personnes qui ont conçu la réforme des programmes rencontre-t-il le point de vue des usagers (futurs enseignants) mais aussi répond-il, peutêtre, à un besoin qui transparait du côté des élèves d'après l'étude que nous avons menée ?

Concrètement, les résultats présentés infra font suite à la passation de 366 questionnaires soumis au printemps 2016 à :

- des futurs enseignants français issus des formations suivantes (161 personnes) :

- Licence 3 en Sciences du langage, parcours Professeur des écoles dans une université parisienne $(31 \mathrm{p}$.)

- Master MEEF $1^{\text {er }}$ degré, $1^{\text {re }}$ année à l'ESPE de Lyon (16 p.)

- Master MEEF $1^{\text {er }}$ degré, $1^{\text {re }}$ année à l'ESPE de Paris (95 p.)

- Master MEEF $2^{\text {nd }}$ degré Lettres modernes, $2^{\mathrm{e}}$ année à l'ESPE de Paris (19 p.)

Futurs enseignants à l'école primaire (FPE)

Futurs enseignants au collège ou au lycée (FPCL)

- des élèves français inscrits dans les niveaux suivants (205 personnes) :

- CE2 dans une école parisienne du $\mathrm{XX}^{\mathrm{e}}$ arrondissement (15 p.)

- CM1-CM2 dans une école parisienne du XX arrondissement (16 p.)

- $\mathrm{CM} 2 \mathrm{a}$ dans une école parisienne du $\mathrm{XX}^{\mathrm{e}}$ arrondissement (22 p.)

CE2

$\mathrm{CM} 2$ 
- CM2a dans une école parisienne du $\mathrm{XX}^{\mathrm{e}}$ arrondissement $(27 \mathrm{p}$.)

- $3^{\mathrm{e}}$ a dans un collège de Nanterre (92) (26 p.)

- $3^{\mathrm{e}} \mathrm{b}$ dans un collège de Nanterre (92) $(25 \mathrm{p}$.)

- $3^{\mathrm{e}} \mathrm{c}$ dans un collège de Nanterre (92) $(22 \mathrm{p}$.)

- $3^{\mathrm{e}}$ a dans un collège à Chartres $(28)(20 \mathrm{p}$.)

- $3^{\mathrm{e}} \mathrm{b}$ dans un collège à Chartres $(28)(17 \mathrm{p}$.)

$\cdot 3^{\mathrm{e}} \mathrm{c}$ dans un collège à Chartres $(28)(15 \mathrm{p}$.)

Les 366 répondants nous apportent un certain éclairage sur les questions posées supra et ce, à plusieurs égards. D'abord, tous les niveaux-clés pour l'étude de la langue sont ici représentés, à la fois au cours de la scolarité obligatoire et dans le cursus de la formation des enseignants. Ensuite, les publics scolaires se montrent plutôt diversifiés - les élèves de $3^{\mathrm{e}}$, par exemple, sont scolarisés pour certains à Nanterre, pour d'autres à Chartres.

L'analyse des résultats livrés par cette enquête nous permettra de questionner d'une part les préjugés intimement liés à l'étude de la langue (est-elle une matière aussi abhorrée qu'on ne l'imagine?), d'interroger d'autre part la maitrise actuelle du COI tant par les enseignants en formation que par les élèves. À l'aide des réponses apportées par ces informateurs à cette dernière question mais aussi à celle de spécifier l'utilité de repérer le(s) COI dans une phrase, nous serons alors amenées à jauger le degré de pertinence de maintenir à ce jour certaines notions grammaticales telles que le COI. Les éléments de réponse fournis par ces quelques questions nous inviteront enfin à revenir sur la problématique plus générale de la disposition des futurs enseignants à accueillir une réforme du discours grammatical aux ambitions simplificatrices et pragmatiques.

\section{Aime-t-on l'étude de la langue?}

Dans un premier temps, nous avons sondé les enseignants en formation et les différents élèves sur leur appréciation de l'étude de la langue en tant que partie d'une discipline scolaire. Il apparait au terme du dépouillement des questionnaires que, quel que soit le niveau d'études, la majorité des informateurs apprécie la grammaire bien que le pourcentage de réponses négatives reste élevé. Ce qui ressort cependant de façon nette, c'est la baisse significative de réponses positives au fur et à mesure que l'on monte dans les niveaux de scolarité obligatoire : en effet, si $67 \%$ des élèves de CE2 apprécient l'étude de la langue, le taux de oui ne s'élève plus qu'à 55\% en CM2 (soit à la fin du cycle 3 en 2008) quand il n'atteint plus que $53 \%$ à la fin du cycle 4 , en classe de $3^{\mathrm{e}}$. Nous remarquons en revanche que si la propension au oui augmente dès lors que l'on considère le pan des enseignants en formation pour enseigner dans les classes du collège (63\% de oui), le taux de réponses affirmatives reste un peu moins élevé du côté des enseignants se destinant à l'école primaire (59\%), constat étonnant au regard de l'orientation des études choisies par certains : un peu plus de $20 \%$ des futurs enseignants, en effet, ont suivi un parcours d'études en Sciences du langage. L'on s'attendait donc à ce que le taux de réponses positives soit supérieur au sein de ces publics ; or, l'augmentation n'est que de l'ordre du $6 \%$ par rapport aux classes de $3^{\mathrm{e}}$. Quant aux informateurs se préparant à l'enseignement aux niveaux du collège ou du lycée, ils ne sont que $63 \%$ à aimer l'étude de la langue, à peine plus que les FPE.

Tableau 1. Pourcentages de réponses par niveaux à la question «Aimez-vous l'étude de la langue ?»

\begin{tabular}{|ccccc|}
\hline CE2 & Oui & Non & Oui \& Non & Abstention \\
\hline CM2 & $67 \%$ & $33 \%$ & $0 \%$ & $0 \%$ \\
$\mathbf{3}^{\mathbf{e}}$ & $55 \%$ & $34 \%$ & $11 \%$ & $0 \%$ \\
\hline
\end{tabular}


Tableau 2. Arguments contre une appréciation positive de l'étude de la langue ${ }^{4}$

\begin{tabular}{|l|c|c|c|c|c|}
\hline & CE2 & $\mathbf{C M 2}$ & $\mathbf{3}^{\text {e }}$ & FPE & FPCL \\
\hline - trop complexe (et jargonnant) & & $12 \%$ & $\mathbf{2 0 \%}$ & $\mathbf{2 8 \%}$ & $\mathbf{8 \%}$ \\
\hline $\begin{array}{l}\text { - trop de règles et d'exceptions, par } \\
\text { cœur }\end{array}$ & $\mathbf{2 0 \%}$ & $\mathbf{1 5 \%}$ & $\mathbf{2 0 \%}$ & $8 \%$ & \\
\hline - ennuyeux, répétitif et peu ludique & $\mathbf{2 7 \%}$ & $9 \%$ & $\mathbf{1 9 \%}$ & $5 \%$ & \\
\hline $\begin{array}{l}\text { - déconnecté des pratiques } \\
\text { quotidiennes }\end{array}$ & & & $5 \%$ & & \\
\hline - fait se sentir nul & $13 \%$ & $3 \%$ & $2 \%$ & $1 \%$ & \\
\hline - inutile & & & $1 \%$ & & \\
\hline - trop abstrait & & & & $4 \%$ & \\
\hline - trop peu sensé & & & & $1 \%$ & \\
\hline - mal présentée, abordée & & & & $1 \%$ & \\
\hline - inintéressant & & & & $1 \%$ & \\
\hline
\end{tabular}

Face à ces premières raisons qui traduisent une certaine aversion pour l'étude de la langue, l'on trouve également d'autres critères, qui vont cette fois dans le sens d'une appréciation générale de cette branche. Parmi les raisons qui sont le souvent évoquées, l'on trouve par exemple les adjectifs intéressant, utile et important, tant dans une perspective (de compréhension ou maitrise) personnelle que sociale.

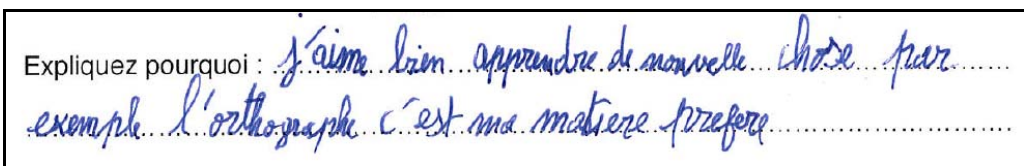

Fig. 6. Justification apportée par un élève de CE2 Expliquez pourquoi : Ore apprend des choses interesente

Fig. 7. Justification apportée par un élève de CM2

S'aprege d'apprendre et de décaqunir flusiour.

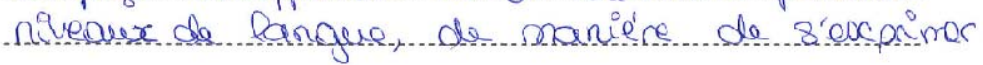

Fig. 8. Justification apportée par un élève de $3^{\mathrm{e}}$

Pour quelles raisons?... L...ét tu de de la langles permat..... d'oppuendre ó mieusc áexprimen

Fig. 9. Justification apportée par un FPE

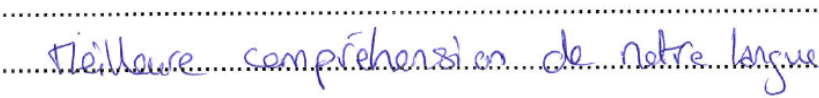

Fig. 10. Justification apportée par un FPCL

Tableau 3. Arguments pour une appréciation positive de l'étude de la langue

\begin{tabular}{|l|c|c|c|c|c|}
\hline Réponses apportées & CE2 & CM2 & $\mathbf{3}^{\text {e }}$ & FPE & FPCL \\
\hline $\begin{array}{l}\text { - intéressant, conduit à de nouvelles } \\
\text { découvertes }\end{array}$ & $\mathbf{5 3 \%}$ & $\mathbf{2 5 \%}$ & $\mathbf{1 9 \%}$ & $11 \%$ & $10 \%$ \\
\hline $\begin{array}{l}\text {-utile pour mieux écrire, lire, parler } \\
\text { utile pour comprendre le fonctionnement } \\
\text { de la langue }\end{array}$ & & $6 \%$ & $\mathbf{1 4 \%}$ & $\mathbf{2 2 \%}$ & $\mathbf{2 1 \%}$ \\
\hline
\end{tabular}




\begin{tabular}{|l|c|c|c|c|c|}
\hline - important (visée sociale, intégration) & & $8 \%$ & $7 \%$ & $2 \%$ & $10 \%$ \\
\hline - facile & $7 \%$ & $3 \%$ & $3 \%$ & & \\
\hline • sacré, objet du patrimoine & & & & $5 \%$ & \\
\hline - logique & & & & $1 \%$ & $15 \%$ \\
\hline - amusant, ludique & & & $1 \%$ & $1 \%$ & \\
\hline
\end{tabular}

Du côté des enseignants en formation, il ressort principalement de l'observation de ces tableaux deux faits; à savoir, pour commencer, que les enseignants en formation reconnaissent pour la plupart l'utilité de développer l'étude de la langue, à des fins d'écriture ou de lecture notamment. Ensuite, que plus d'un quart des futurs enseignants du primaire estime que l'étude de la langue reste une branche de la discipline complexe et faisant appel à une terminologie spécifique dense, dont l'utilité ne peut être que questionnée. C'est à cette fin que nous avons ensuite interrogé les différents publics sur le cas particulier du COI, notion grammaticale choisie parmi d'autres en ce qu'elle illustre le problème plus général rencontré par la grammaire scolaire contemporaine.

\section{Le cas particulier du « COI »}

Les programmes scolaires français en vigueur depuis 2008 fixent l'apprentissage de la notion du COI ainsi que la maitrise de sa reconnaissance dans une phrase, au cycle 3 (CE2CM1-CM2 ; [13], p. 21). Bien qu'elles entendent alors « favoriser la compréhension des textes lus et entendus» ([13], p. 22) et «améliorer l'expression en vue d'en garantir la justesse, la correction syntaxique et orthographique » (ibid.), les prescriptions ministérielles pour le cycle 3 insistent parallèlement sur l'" acqui[sition] progressiv[e] [du] vocabulaire grammatical qui se rapporte aux notions étudiées » (ibid.), parmi lesquelles le COI. L'on s'attend en conséquence à ce que la notion grammaticale du COI soit parfaitement acquise et maitrisée à la fin de la CM2 comme aux niveaux suivants, quand on admettrait cependant, en regard de sa seule découverte à l'âge de 8 ou 9 ans, qu'elle soit moins bien manipulée en classe de CE2. Les résultats livrés par l'enquête menée montrent néanmoins que la progression imaginée n'est pas aussi remarquable qu'espérée. En effet, bien que la théorie $^{5}$ paraisse maitrisée à $79 \%$ dès le CE2, ce taux reste pour ainsi dire inchangé jusqu'en classe de $3^{\mathrm{e}}$, où le pourcentage s'élève à $78 \%$. La véritable augmentation est remarquée du côté des enseignants en formation, avec des valeurs qui atteignent les $91 \%$. $A$ contrario, on remarque que la reconnaissance d'un COI dans une phrase ne va pas de soi, et il parait difficile de corréler la maitrise de son repérage dans une phrase à l'acquisition des connaissances théoriques ${ }^{6}$. Le travail d'identification du COI dans une phrase semble d'ailleurs poser davantage de problèmes aux élèves de CM2 qu'aux élèves de CE2 - même si la différence entre les deux pourcentages est minime -, ce qui pourrait s'expliquer par le fait que les élèves de CE2 ayant répondu au questionnaire venaient d'étudier ce point de grammaire. Si une amélioration est constatée avec les $3^{\mathrm{e}}(64 \%)$, l'augmentation est plus nette du côté des enseignants en formation, qu'ils se destinent à l'enseignement à l'école primaire ou aux collège et lycée. L'on ne peut que constater cependant que le taux de $100 \%$ qui devrait être atteint, idéalement, par les enseignants de demain, ne l'est pas : la moyenne obtenue au test de reconnaissance des COI dans sept phrases proposées (cf. infra) oscille autour des $94 \%$.

Tableau 4. Pourcentages, par année, des maitrises théorique et pratique de la notion de COI

\begin{tabular}{|c|c|c|}
\hline & Théorie & Pratique \\
\hline CE2 & $79 \%$ & $58 \%$ \\
CM2 & $80 \%$ & $55 \%$ \\
\hline
\end{tabular}




\begin{tabular}{ccc}
$\mathbf{3}^{\mathbf{e}}$ & $78 \%$ & $64 \%$ \\
FPE & $92 \%$ & $89 \%$ \\
FPCL & $91 \%$ & $94 \%$ \\
\hline
\end{tabular}

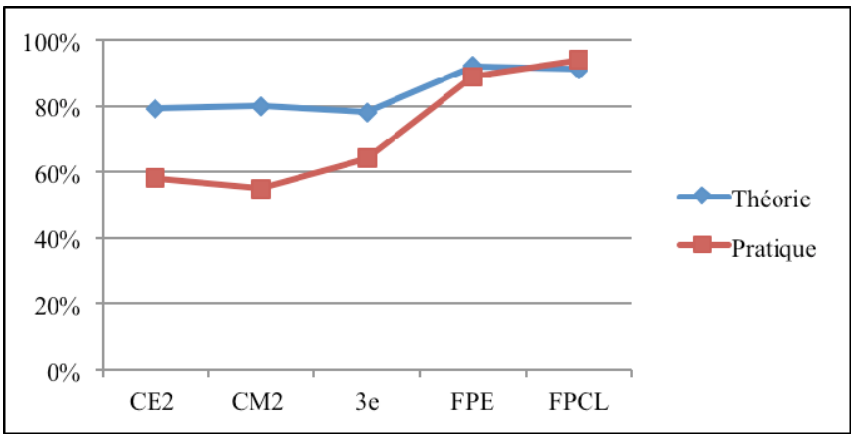

Fig. 11. Progression, par année, des maitrises théorique et pratique de la notion de COI

Il s'avère donc que, de façon générale, la notion de COI est relativement bien acquise (théorie) et maitrisée (pratique) par les enseignants en formation, même si elle ne l'est pas pleinement, ce qui est pourtant attendu d'un futur enseignant, qu'il ait déjà passé le concours (fig. 14-15) ou non (fig. 12-13).

3) Cochez les phrases qui ont un $\mathrm{COI}$ et soulignez-le dans la phrase.

$\square$ Julien attend son frère.

$\square$ Le jeune homme succédera à son père l'an prochain.

$\otimes$ Pense aux sans abris.

G Juliette va à la piscine le mercredi matin.

[7 II se souvient de sa première bicyclette.

Les enfants se donnent la main.

$\square$ J'y pense.

Fig. 12. Réponses apportées par un FPE

$\square$ Julien attend son frère.

Le jeune homme succédera à son père l'an prochain.

$\forall$ Pense aux sans abris.

$\square$ Juliette va à la piscine le mercredi matin.

$凶$ II se souvient de sa première bicyclette.

$\square$ Les enfants se donnent la main.

$\square$ J'y pense.

Fig. 13. Réponses apportées par un FPE

$\square$ Julien attend son frère.

丈. Le jeune homme succédera à son père l'an prochain.

$\forall$ Pense aux sans abris.

@uliette va à la piscine le mercredi matin.

XII se souvient de sa première bicyclette.

$\square$ Les enfants se donnent la main.

\'y pense.

Fig. 14. Réponses apportées par un FPCL 


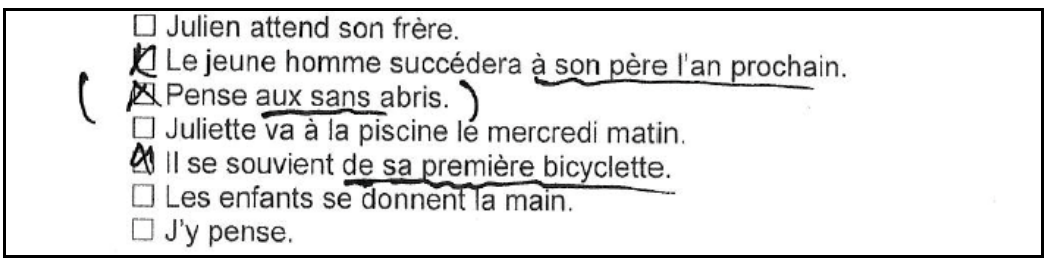

Fig. 15. Réponses apportées par un FPCL

Parallèlement à ces constats, l'on est en droit de s'interroger sur les fins de l'enseignement de cette notion grammaticale, tant dans une perspective théorique que pratique (travail d'identification). La question suivante du questionnaire entendait précisément sonder les élèves et les enseignants en formation sur l'utilité « de chercher à identifier le COI dans une phrase ». Les réponses obtenues abondent majoritairement dans un sens : «à rien » ou, plus humblement, «je ne sais pas » sont en effet les réponses les plus fréquemment apportées, quel que soit le niveau d'étude observé. À la différence des élèves du primaire, cependant, l'on constate aussi qu'une autre réponse est régulièrement évoquée par les futurs enseignants : plutôt d'ordre sémantique et relatif à la phrase $(22 \%$, déjà annoncée d'ailleurs par les réponses des élèves de $3^{\mathrm{e}}$ ) voire sémantico-syntaxique et relatif au verbe $(20 \%)$ pour les FPE ; plutôt syntaxique et fonctionnel, intimement lié au cadre de la phrase $(42 \%)$ pour les FPCL.

Tableau 5. Réponses à la question de l'utilité de chercher à identifier le COI

\begin{tabular}{|c|c|c|c|c|c|}
\hline Réponses apportées & CE2 & CM2 & $3^{\mathrm{e}}$ & FPE & FPCL \\
\hline - à rien, je ne sais pas & $60 \%$ & $25 \%$ & $57 \%$ & $32 \%$ & $37 \%$ \\
\hline - comprendre le sens d'une phrase & & & $24 \%$ & $22 \%$ & $5 \%$ \\
\hline $\begin{array}{l}\text { - comprendre la structure/le fonctionnement } \\
\text { d'une phrase }\end{array}$ & & & $3 \%$ & $20 \%$ & $42 \%$ \\
\hline - compléter le verbe & & $2 \%$ & $3 \%$ & $20 \%$ & $26 \%$ \\
\hline - identifier de qui/quoi l'on parle & $7 \%$ & $9 \%$ & $8 \%$ & $1 \%$ & \\
\hline $\begin{array}{l}\text { - à le distinguer du COD (visée généralement } \\
\text { orthographique) }\end{array}$ & & $8 \%$ & $1 \%$ & $7 \%$ & $5 \%$ \\
\hline - à différencier les verbes transitifs et intransitifs & & & & $6 \%$ & \\
\hline $\begin{array}{l}\text {-à ne pas le confondre avec les autres } \\
\text { compléments (du nom, circonstanciels) }\end{array}$ & & $5 \%$ & $3 \%$ & $2 \%$ & $11 \%$ \\
\hline - à voir si le COI est dans la phrase ou pas & $7 \%$ & $2 \%$ & $1 \%$ & & \\
\hline • à mieux écrire, s'exprimer & $7 \%$ & $5 \%$ & $1 \%$ & $1 \%$ & \\
\hline - comprendre la pronominalisation & & & & $1 \%$ & \\
\hline - à avoir une bonne note au contrôle & & $8 \%$ & $1 \%$ & & \\
\hline - parce que la maitresse est obligée d'en parler & & $2 \%$ & & & \\
\hline
\end{tabular}

4) Selon vous, à quoi cela sert-il de chercher à identifier le "COI " dans une phrase?

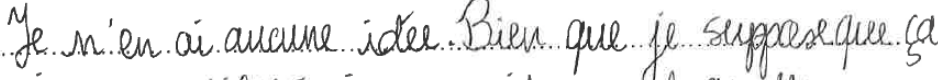 di une utilité je fue... voit pas laquelle

Fig. 16. Réponse apportée par un élève de 3 


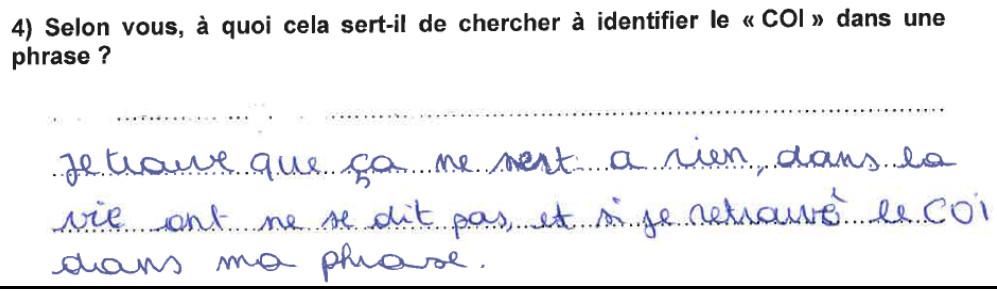

Fig. 17. Réponse apportée par un élève de $3^{\mathrm{e}}$

Au demeurant, certaines réponses apportées par les élèves de CM2 sont surprenantes : on relèvera par exemple les mentions " avoir une bonne note au contrôle » $(8 \%)$ ou " parce la maitresse est obligée d'en parler» $(2 \%)$. Outre les réponses aussi utilitaristes que sincères (i.e. avoir une bonne note, mieux écrire), il ressort du tableau 5 une certaine récurrence dans les réponses fournies : le critère du sens est régulièrement évoqué, bien que l'on puisse douter de sa pertinence en raison du fait que la phrase fait sens, en principe, indépendamment de la tâche de reconnaissance d'un quelconque COI en son sein. L'identification d'un COI dans une phrase apparait donc en dernier recours, au vu des réponses données, comme outil pour une meilleure compréhension de la structure ou du fonctionnement de la langue, pensée qui commence à émerger en classe de $3^{\mathrm{e}}$ sans pour autant s'imposer (3\%) alors que, dans une optique de progression scolaire, la classe de $3^{\mathrm{e}}$ signe la fin de l'étude de la langue (grammaire). Cela donne à réfléchir sur l'utilité du discours grammatical telle qu'elle est imaginée par les élèves au terme de leur parcours scolaire.

Les futurs enseignants sont donc les seuls à véritablement penser en termes d'analyse fonctionnelle de la phrase et en termes d'accord du participe passé (d'où la nécessité de distinguer COD et COI). De même, ils sont les seuls à pointer le sujet de la transitivité verbale $(6 \%)$ ou le test de la pronominalisation utilisé $(1 \%$; quoique, ici, la réponse est maladroitement formulée par le FPE). En réalité, ces différentes entrées procèdent selon nous d'une seule et même cause : la volonté de justifier l'acquisition et la maitrise d'une nomenclature grammaticale par, tautologiquement, le recours aux notions et tests syntaxiques qui en découlent. C'est ainsi que la transitivité est le fruit de la présence de $\mathrm{COD} / \mathrm{COI}$, que la pronominalisation est évoquée dans le but d'identifier le COD/COI. En conséquence, a-t-on vraiment besoin des notions de COD et de COI aujourd'hui ? À plus forte raison, est-il nécessaire de les enseigner?

\section{Degrés de pertinence de la notion de COI aujourd'hui}

Selon nous, la réponse à la question précédente mérite d'être évaluée à l'aune de son histoire. Comme nous l'avons rappelé, la notion de COI est le résultat d'une construction historique, fruit du datif qui, en latin classique, était un cas distinct de l'accusatif. Aujourd'hui, l'identification du COD est n'est plus qu'intimement liée à des questions d'orthographe grammaticale (accord du participe passé employé avec avoir si le COD précède). Ce rôle est pourtant inconnu du COI, ce qui ne peut qu'inviter à questionner la légitimité de son repérage dans la phrase à l'école. C'est en ce sens qu'abonde précisément le B.O. du 26 novembre 2015 [16], lequel ne propose plus de distinction entre les différents compléments du verbe (cf. supra). Il paraitrait donc plausible de se passer de cette distinction terminologique. L'analyse grammaticale proposée par les nouvelles prescriptions scolaires se trouverait ainsi délestée d'une partie d'un bagage historique sans conséquence immédiate pour la correction de l'écriture ${ }^{7}$ ou la maitrise de la langue.

Cela étant, et cela s'apparente à un second argument en faveur de la fusion des deux compléments sous l'appellation de compléments $d u$ verbe, le B.O. soutient qu'une seule distinction fondamentale doit être opérée : compléments du verbe vs compléments de 
phrase (CP). Pour ce faire, les techniques listées pour reconnaitre les deux types de compléments sont au nombre de trois dès le cycle 3 : la suppression, le déplacement en tête de phrase et la pronominalisation ([16], p. 118). Cependant, force est de constater que chacun de ces trois tests s'accompagne d'inconvénients, pour certains majeurs. Considérons les exemples suivants qui en présentent les limites :

(a) suppression :

- COI : Jean a donné son manteau à Paul. > Jean a donné son manteau.

- CP : Ce soir, Jean sort. > Jean sort.

(b) déplacement :

- COI : À son père il racontait les choses tristes. (cf. [18])

- CP : À la tombée de la nuit, il devra être rentré.

(c) pronominalisation :

- COI : Il parle à Marie. > Il lui parle.

- CP : Il joue au ballon sur la plage. > Il y joue au ballon.

Les trois manipulations syntaxiques se montrant insuffisantes, il nous faut identifier un autre argument pour discriminer les compléments du verbe et de phrase. Le test de la négation pourrait selon nous s'offrir comme une alternative potentielle. Le test de la négation, en effet, permet de voir si un élément est intégré au prédicat (complément du verbe) ou en réchappe (complément de phrase) : dans une phrase comportant une négation descriptive (cf. [19]), si un terme est nié par cette négation, alors il sera identifié comme un complément de verbe ; s'il y échappe, c'est qu'il s'agit d'un complément de phrase ([20], p. 86, 189). Dans les exemples précédents, en effet, le test de la négation permet selon nous de différencier plus clairement les COI des CP :

- COI : Jean n'a pas donné son manteau à Paul.

- $\mathrm{COI}$ : À son père il ne racontait pas les choses tristes.

- COI : Il ne lui parle pas.

- CP : Ce soir, Jean ne sort pas.

- $\mathrm{CP}$ : A la tombée de la nuit, il ne devra pas être rentré.

- $\mathrm{CP}$ : Il ne joue pas au ballon sur la plage.

Les résultats présentés par le test sont moins transparents dès lors que le complément prétendument de phrase se trouve directement placé après le verbe : Il ne mange pas au restaurant (deux lectures possibles: (a) il mange, mais ailleurs; (b) il est dans le restaurant mais il ne mange pas). Cela est dû au caractère problématique de l'étendue du prédicat: un complément pensé "de phrase » niable par une négation descriptive se révèlera en fait être un complément intégré au prédicat, c'est-à-dire un complément « $d u$ verbe ». En ce sens, les réformes de 2016 [16] ne sont peut-être pas aussi abouties qu'elles le laissent à penser: indépendamment des trois manipulations syntaxiques citées (suppression, déplacement, pronominalisation), c'est l'articulation même complément de phrase - complément du verbe qu'il convient de repenser. Autrement dit, un certain nombre de compléments admis « de phrase » par la grammaire scolaire ne le sont pas; ce sont en réalité des compléments du verbe.

Le test de la négation n'est pas mentionné explicitement dans le B.O. de 2015 [16], mais il pourrait être entendu à travers la mention "manipulations syntaxiques pour mettre en évidence les constructions verbales » (p. 242). C'est donc à cette manipulation spécifique, ainsi qu'à la claire dissociation entre les compléments de phrase et de verbe qu'il faudrait selon nous former les enseignants.

En guise de troisième et dernier argument, il nous faut rappeler que lorsqu'un locuteur s'exprime (à l'oral comme à l'écrit), il produit spontanément des COI ; il n'analyse pas pour autant sa phrase en ces termes. En ce sens, l'on ne peut qu'interroger le bienfondé des activités de repérage et d'étiquetage gratuites. Peut-être serait-il d'ailleurs bénéfique d'en faire prendre conscience aux (futurs) enseignants. 


\section{Réformer le discours grammatical ?}

Nous avons finalement interrogé les futurs enseignants sur l'accueil qu'ils réservent à la nouvelle terminologie grammaticale relative aux compléments du verbe. On constate que les néo-enseignants (M2) sont les plus enclins à conserver la distinction COD / COI (74\%), alors qu'il n'y a que $35 \%$ des étudiants de L3 à Paris qui sont favorables à un tel maintien métalinguistique. Pour les futurs enseignants français restants (M1), le pourcentage est de $65 \%$. Les différences notables à ces trois niveaux de formation se justifient peut-être par les enseignements dont ont bénéficié les trois publics. Alors que les étudiants de M2 sont déjà en poste et ont suivi une formation traditionnelle, les étudiants de M1 commencent à être sensibilisés aux contenus de la réforme, quand les futurs PE en L3 sont plus ouverts à un discours (ré)formateur au regard de la terminologie, eu égard sans doute aux premiers pas qu'ils font dans le domaine de l'enseignement.

Tableau 6. Réponses à la question «Est-il pertinent de distinguer le COD » du COI ?

\begin{tabular}{|ccccc|}
\hline & Oui & Non & Oui \& non & o \\
\hline L3 Paris & $35 \%$ & $\mathbf{4 8 \%}$ & $6 \%$ & $10 \%$ \\
M1 Lyon \& Paris & $\mathbf{6 5 \%}$ & $17 \%$ & $5 \%$ & $13 \%$ \\
M2 Paris & $\mathbf{7 4 \%}$ & $5 \%$ & $5 \%$ & $16 \%$ \\
\hline
\end{tabular}

Les résultats obtenus dans le tableau 7 corroborent la plupart des premières hypothèses émises supra : en écho aux résultats précédents, les étudiants de L3 sont majoritairement favorables à une simplification de la terminologie grammaticale. Les M1 semblent plus mitigés en ce qu'ils sont $54 \%$ à être réfractaires à la seule appellation de Complément $d u$ verbe, position qui va à l'encontre des prescriptions ministérielles du 26 novembre 2015. Quant aux étudiants de M2, le nombre de non approche par son taux de $58 \%$ celui donné par les M1 (54\%) tandis que le nombre de réponses affirmatives $(32 \%)$ chute de façon notable par rapport à ces mêmes étudiants de master $1(41 \%)$. Il nous semble cependant utile de souligner le fait que $11 \%$ des réponses balancent entre le oui et le non, ces étudiants étayant leur réponse par un commentaire réflexif sur les atouts et inconvénients de la réforme en préparation ${ }^{8}$.

Tableau 7. Réponses à la question «Êtes-vous favorable à la seule appellation Compléments du verbe?»

\begin{tabular}{|ccccc|}
\hline & Oui & Non & Oui \& non & क \\
\hline L3 Paris & $\mathbf{8 1 \%}$ & $13 \%$ & $6 \%$ & $0 \%$ \\
M1 Lyon \& Paris & $\mathbf{4 1 \%}$ & $\mathbf{5 4 \%}$ & $2 \%$ & $4 \%$ \\
M2 Paris & $32 \%$ & $\mathbf{5 8 \%}$ & $11 \%$ & $0 \%$ \\
\hline
\end{tabular}

Enfin, les critères avancés de part et d'autre contre une fusion terminologique générique peuvent surprendre par leur proximité avec les arguments généralement avancés contre une réforme de l'orthographe. C'est ainsi que les motifs du nivèlement par le bas et de l'appauvrissement de la langue, de l'habitude ou encore de l'héritage reviennent souvent sur le devant de la scène. A contrario, c'est davantage la simplification du discours grammatical et le fait de libérer du temps en classe au profit d'autres activités qui attirent les faveurs de certains enseignants en formation, les incitant à marcher dans le sens de la réforme. C'est en cela que l'on assiste à l'émergence d'un terreau fertile, favorable à une réflexion sur le choix des contenus à transposer didactiquement, ficelle à tirer pour que, dans les années à venir, la grammaire travaillée à l'école regagne définitivement en sens. 


\section{Conclusion}

Que retenir des résultats discutés à l'instant? Il ressort de prime abord que serait profitable une sensibilisation des enseignants au fait que la simplification de la terminologie n'est pas inéluctablement un nivèlement par le bas, mais qu'à cette réforme répond une véritable réorganisation du système grammatical enseigné en milieu scolaire. Le traitement des compléments du verbe $v s$ de phrase nous semble s'en faire l'illustration. Ensuite, étant donné que les objectifs de formation des élèves à l'étude de la langue ne sont plus ceux du simple repérage-étiquetage mais de la «compréhension synthétique du système de la langue » et de son "organisation» ([16], p. 229, 238), il serait sans doute souhaitable d'initier les enseignants à la (re)manipulation de la langue : l'idée est de (se laisser) déstabiliser pour parvenir à mieux saisir le fonctionnement linguistique et à élaborer progressivement la notion de degré d'acceptabilité avec les élèves.

\section{Références}

1. B. Bouard, « De l'apport de l'histoire dans l'enseignement de la langue. L'exemple des compléments du verbe », Le français aujourd'hui, 192, 15-32 (2016).

2. S. Piron, «La grammaire du français au XVIII ${ }^{\mathrm{e}}$ siècle. $2^{\mathrm{e}}$ partie », Correspondance, 14.3, en ligne (2009).

3. C. Buffier, Grammaire françoise sur un plan nouveau, Paris, N. le Clerc (1709).

4. B. Bouard, «L'émergence de la notion de « complément » est-elle une invention ou une innovation? », in Neveu F. \& al. (dir), Actes du $1^{\text {er }}$ CMLF, Paris, ILF, en ligne (2008).

5. P. Restaut, Principes généraux et raisonnés de la grammaire françoise, Paris, J. Desaint (1730).

6. C.-F. Lhomond, Elémens de la grammaire françoise, Paris, Colas (1780).

7. S. Piron, « La grammaire du français au XIX ${ }^{\mathrm{e}}$ siècle. $1^{\text {re }}$ partie », Correspondance, 14.4, en ligne (2009).

8. F.-J.-M. Noël, C.-P. Chapsal, Nouvelle grammaire française, sur un plan trèsméthodique, avec de nombreux exercices d'orthographe, de syntaxe et de ponctuation [...], $30^{\mathrm{e}}$ éd., Paris, Maire Nyon (1837).

9. A. Chervel, Histoire de la grammaire scolaire... et il fallut apprendre à écrire à tous les petits Français, Paris, Payot (1977).

10. S. Piron, «La grammaire du français au XIX ${ }^{\mathrm{e}}$ siècle. $2^{\mathrm{e}}$ partie », Correspondance, 15.1, en ligne (2009).

11. C.-P. Girault-Duvivier, «Grammaire des grammaires ou analyse raisonnée des meilleurs traités sur la langue française », Bruxelles/Paris, P.-J. de Mat/Janet et Cotelle (1811).

12. S. Piron, «La grammaire du français au $\mathrm{XX}^{\mathrm{e}}$ siècle. $1^{\mathrm{re}}$ partie », Correspondance, 15/4, en ligne (2010).

13. Ministère de l'Éducation nationale, Horaires et programmes d'enseignement de l'école primaire, Bulletin officiel hors série ${ }^{\circ} 3$, France (juin 2008).

14. Ministère de l'Éducation nationale, Programmes du collège. Programme de l'enseignement de français, Bulletin officiel hors série $n^{\circ} 6$, France (aout 2008).

15. Ministère de l'Éducation nationale, Terminologie grammaticale, Centre National de Documentation Pédagogique, France (1997).

16. Ministère de l'Éducation nationale, Programmes d'enseignement du cycle des apprentissages fondamentaux (cycle 2), du cycle de consolidation (cycle 3) et du cycle 
des approfondissements (cycle 4), Bulletin officiel spécial $n^{\circ} 10$, France (novembre 2015).

17. C. Cherdon, B. Wautelet, Bingo !, Bruxelles, De Boeck (2014).

18. F. Sabio, «L'antéposition des compléments dans le français contemporain. L'exemple des objets directs », Lingvisticae Investigationes, 29/1, 173-182 (2006).

19. O. Ducrot, La preuve et le dire, Paris, Mame (1973).

20.D. Van Raemdonck, Le sens grammatical. Référentiel à l'usage des enseignants, Bruxelles, PIE Peter Lang (2011).

${ }^{1}$ Année choisie parce qu'elle signe la fin de l'enseignement à l'école primaire [13, 16].

${ }^{2}$ Année choisie parce qu'elle signe la fin du cycle 4 d'après les B.O. de 2008 [14] et de 2016 [16].

${ }^{3}$ Nous tenons à remercier chaleureusement Félicie Cazaubon et Anaïs Daudigny d'avoir initié cette étude avec une classe de CM2. Nous remercions également l'ensemble des enseignants et étudiants qui ont accepté de se soumettre/répondre à ce questionnaire.

${ }^{4}$ Les catégories données dans le tableau procèdent de notre synthèse des différentes réponses apportées par les enquêtés.

${ }^{5}$ Nous entendons ici par théorie la capacité de la personne interrogée à définir ou à associer un contenu notionnel au COI tandis que la maitrise réfère à sa capacité à repérer un $\mathrm{COI}$ dans un énoncé donné.

${ }^{6}$ Par «maitrise » nous entendons la capacité de l'élève à identifier le COI dans une phrase. Nous entendons en revanche par " acquisition des connaissances théoriques », l'aptitude de l'élève à définir la notion même de "COI ", à préciser ses contours théoriques par, notamment, l'inventaire de ses propriétés. Dans le tableau 4, l'entrée « théorie » renvoie donc aux connaissances théoriques (révélées par les réponses données à la question 2) tandis que le volet pratique réfère à la capacité de repérer un COI dans une phrase (cf. les réponses données à la question 3). En ce qui concerne les pourcentages, nous les avons établis de la façon suivante : pour la question 2 comme la question 3, nous avons observé le nombre de réponses correctes et avons donné, à partir de là, un score sur 6 (Q2) et sur 7 (Q3), équivalent à $100 \%$ dans le cas où toutes les réponses sont bonnes. Après avoir fait cela pour les 366 questionnaires récoltés, nous avons calculé les pourcentages finaux correspondants.

${ }^{7}$ Pour la question plus vaste de l'accord du participe passé, il est possible de faire l'économie totale du métalangage. Cf. par exemple la méthode proposée par Cherdon \& Wautelet en 2014 [17] : Règle. Je m'arrête après le participe passé, et je pose la question :

Est-ce que je sais QUI/CE QUI EST + participe passé?

Si je réponds NON, le participe passé ne s'accorde pas.

$\mathrm{Si}$ je réponds OUI, le participe passé s'accorde en genre et en nombre avec le nom ou le pronom auquel il se rapporte.

${ }^{8}$ À ce propos, nous remercions l'un de nos deux relecteurs d'avoir soulevé la question suivante : «Y a-t-il des corrélations intéressantes entre les réponses à la question sur les attitudes (Tableau 1/Fig.1) et les réponses aux questions sur la réforme du discours (Tableaux $6 \&$ 7) ? » Les pourcentages obtenus n'apportent pas de réponse claire à cette question qui mériterait cependant une étude spécifique, amorcée par ailleurs dans le cadre du projet de recherche-action «Manipuler la langue pour comprendre, apprendre et écrire ». 